\title{
Editorial
}

\section{Novel Photomedicine}

\author{
Victor Loschenov, ${ }^{1,2}$ Rudolf Steiner, ${ }^{3}$ Alexander Potapov, ${ }^{4}$ and Alexander Douplik ${ }^{5}$ \\ ${ }^{1}$ M. Prokhorov General Physics Institute of the Russian Academy of Sciences, Vavilov Street 38, Moscow 119991, Russia \\ ${ }^{2}$ National Research Nuclear University MEPhI (Moscow Engineering Physics Institute), Kashirskoe Shosse 31, Moscow 115409, Russia \\ ${ }^{3}$ Institut fuer Lasertechnologien in der Medizin und Messtechnik an der Universität Ulm, Helmholtz Street 12, 89081 Ulm, Germany \\ ${ }^{4}$ Burdenko Neurosurgery Institute, 4th Tverskaya-Yamskaya Street 16, Moscow 125047, Russia \\ ${ }^{5}$ Department of Physics, Ryerson University, 350 Victoria Street, Toronto, ON, Canada M5B 2K3
}

Correspondence should be addressed to Victor Loschenov; loschenov@mail.ru

Received 3 September 2014; Accepted 3 September 2014; Published 31 December 2014

Copyright (c) 2014 Victor Loschenov et al. This is an open access article distributed under the Creative Commons Attribution License, which permits unrestricted use, distribution, and reproduction in any medium, provided the original work is properly cited.

In the past years physicist, physicians, chemists, biologists, and other scientists are cooperating in developing new methods in treatment and diagnosis of a huge problem of nowadays-malignant lesions. The scientists are elaborating new methods, novel agents, improving existing methods of diagnosis and treatment of wide classes and types of cancers. Photomedicine - is a huge domain covering a wide area from diagnosis to treatment of a large spectrum of malignancies, from developing of new agents to improvement of already existing technologies. The special issue Novel Photomedicine highlights the recent advances and last approaches in optogenetics, fluorescence diagnosis, and photodynamic therapy and moreover suggests the post procedures to avoid sideeffects of the photodynamic therapy. We give a short overview of the published articles below.

The review article "Optogenetics: Novel Tools for Controlling Mammalian Cell Functions with Light” is representing the recent approaches in optogenetics: techniques, methods, and so forth. The authors are primarily concentrating on the most commonly used photosensitive protein, channelrhodopsin protein. The most recently developed tools are also described. Undoubtedly, optogenetics is a fast developing tool with perspective future, although it appeared in recent decade.

An interesting approach of using microbubbles for enhancing optical properties was represented in paper entitled "Optical Scattering Properties of Intralipid Phantom in Presence of Encapsulated Microbubbles." The ability of the microbubbles as optical contrast agents is investigated in variety of intralipid phantoms. The results are showing the future potential of the encapsulated microbubbles in optical diagnosis, as they effectively change optical properties of the intralipid phantoms, which could help to improve the optical imaging of malignant tissues.

A promising mechanism for photodynamic therapy was described and investigated in "Autoregulatory Feedback Mechanism of P38MAPK/Caspase- 8 in Photodynamic Therapy-hydrophilic/Lipophilic Tetra- $\alpha$-(4-carboxyphenoxy) Phthalocyanine Zinc-Induced Apoptosis of Human Hepatocellular cCarcinoma Bel-7402 Cells." The authors firstly introduce the effect of T-alpha PCZn-PDT on apoptosis of human hepatocellular carcinoma cells with and without inhibitors, as well as on Caspase-3, Bid, Cytochrome c, and mitochondria membrane potential, respectively. Their investigation demonstrates that Caspase-3, Bcl-2, Bid, and mitochondria are involved in autoregulatory feedback of P38MAPK/Caspase-8 during photodynamic therapy of abovementioned cells.

The most recent problems and solutions for most common malignancy are reviewed in the paper "Overview on Topical 5-ALA Photodynamic Therapy use for Nonmelanoma skin cancers." The review includes history of photodynamic therapy, procedure itself, and especially treatment of nonmelanoma skin cancer. Afterwards the postprocedure such as photorejuvenation is discussed, and new concepts for illumination and limits of application are widely highlighted.

A contribution to improve fluorescence diagnosis was represented in "Scattered and Fluorescent Photon Track Reconstruction in a Biological Tissue." The paper is considering the way laser-induced fluorescence spreads in tissue. The 
authors firstly suggest and calculate an effective fluorescence anisotropy factor on example of brain tissue phantoms. The obtained result allows simplifying the process of fluorescence probing depth's determination in order to improve analysis of deep tissue regions during neurosurgical operations.

A variety of forward scattering quasi 1-D paths are discussed in the paper "Reflectance of Biological Turbid Tissues under Wide Area Illumination: Single Backward Scattering Approach." The contribution of single backward scattering to reflectance spectra is investigated and its potential effectiveness for the distances between transport and reduced scattering domain is argued. This remarkable achievement allows taking into account not only mismatched boundary conditions but also multilayer geometry and moreover can be used for real-time spectral processing.

A way to increase photodynamic efficiency is reflected in "Multiorganelle Localization of Metallated Phthalocyanine Photosensitizer in Colorectal Cancer Cells (DLD-1 and CaCo-2) Enhances Efficacy of Photodynamic Therapy." The authors' approach was in increasing the apoptotic cell death during PDT treatment of colorectal cancer on the example of two cancer cell lines (DLD-1 and CaCo-2). After 1 and $24 \mathrm{~h}$ there was a significant increase in both cathepsin D and cytochrome C. They state that the photosensitiser leads to different response from cells most probably according to the stage of the tumorogenesis. Although the way photosensitiser induces the cell death has to be studied further, it is hypothesized that sulfonated zinc phthalocyanine photosensitiser is capable of inducing apoptotic cell death that is promoted by lysosomal photooxidation.

A method of visualisation of the diffuse reflected light is described in research entitled "Two-Stage Analysis on Models for Quantitative Differentiation of Early-Pathological Bladder States." A simulation method is considering 5 states of epithelium tissue, from normal to precancerous. The authors found the influence of optical properties change on diffuse-reflectance signal distributions on the surface of the multilayered phantoms. The analysis is made on different threshold levels and could be used as a base for inverse problem solution to interpret diffuse-reflectance signals from epithelial tissues, although there were no additional markers used.

A residual effect of PDT treatment is investigated in a paper entitled "Photoprotective Effect of The Plant Collaea Argentina against Adverse Effects Induced by Photodynamic Therapy." The authors proposed to use a special agentthe methanolic extract of the Collaea argentina-to protect healthy cells from side-effects of PDT with different types of photosensitisers in different concentrations. However the additional research with in vivo experiments and isolation of the compound responsible for the photoprotection has to be conducted; it is already stated that the extract interferes with singlet oxygen production during photodynamic treatment, which is a promising result to protect skin from PDT-induced photodamage of healthy skin.

Another research is dealing with PDT safety to nondamaged tissue and entitled "Safety Study of Photodynamic Therapy Using Talaporfin Sodium in the Pancreas and Surrounding Tissues in the Syrian Golden Hamster." The authors investigated the ability of PDT with talaporfin sodium (TS) to treat pancreas without damaging surrounding tissues. Although minimisation of the exposure of the duodenum to light is required, the conducted research showed that PDT with TS in Phase 1 studies of PDT for pancreatic cancer in inoperable patients with localised disease is supported.

A new promising agent for PDT is announced in "Potential Use of C60/2-Hydroxypropyl- $\beta$-Cyclodextrin Nanoparticles as a New Photosensitizer in the Treatment of Cancer." The paper is studying photosensitizing abilities of the nanoparticles by means of electron spin resonance and phototoxicity on cancer cells, the photoinduced antitumor effect was evaluated as well. However accumulation of the nanoparticles and pharmacokinetics of the process after injection is still under investigation; the authors obtained the remarkable effect of tumor growth suppression and found the difference between light doses provided to tumor.

Another group presented an improvement of colon cancer diagnosis in the paper entitled "Fluorescence Diagnostics of Colon Malignant and Premalignant Lesions Using 5Aminolevulinic Acid." The research was based on 5-ALA effectiveness for fluorescence diagnosis of colon polyps. Local fluorescent diagnosis as an additional step in combined approach during colonoscopy allowed for significantly increasing specificity from $62.5 \%$ to $93.7 \%$. Moreover the method applied allowed targeting biopsy as well as identifying malignant transformations in colon.

\section{Acknowledgment}

The group of editors would like to express their thankfulness to all the contributing authors for submitting their valuable and informative manuscripts. We would like to wish all the authors success in their researches. 

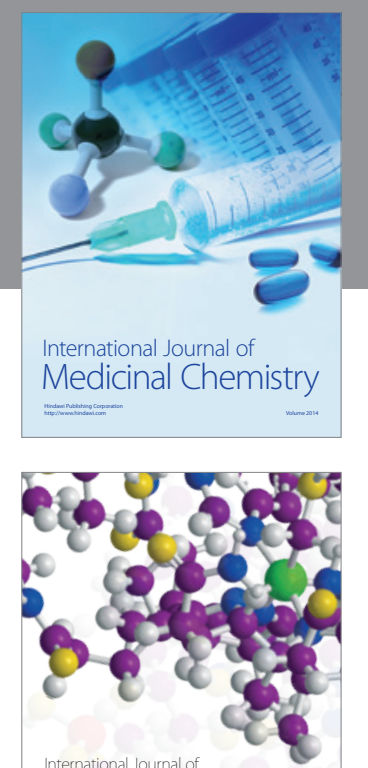

\section{Carbohydrate} Chemistry

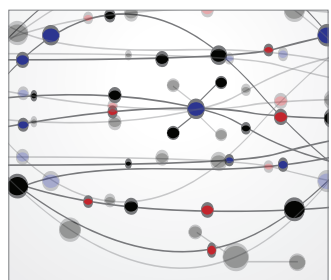

The Scientific World Journal
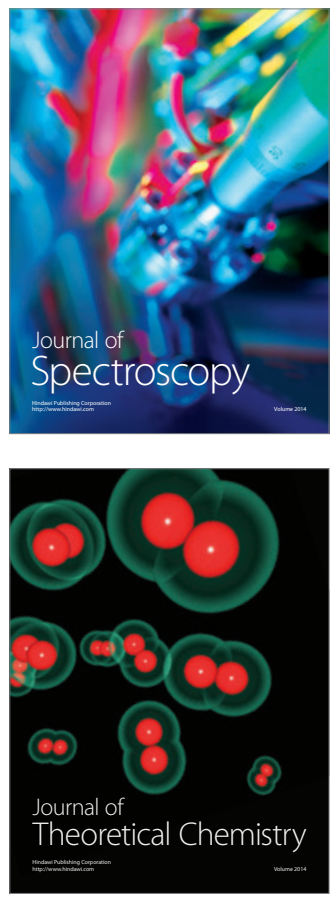
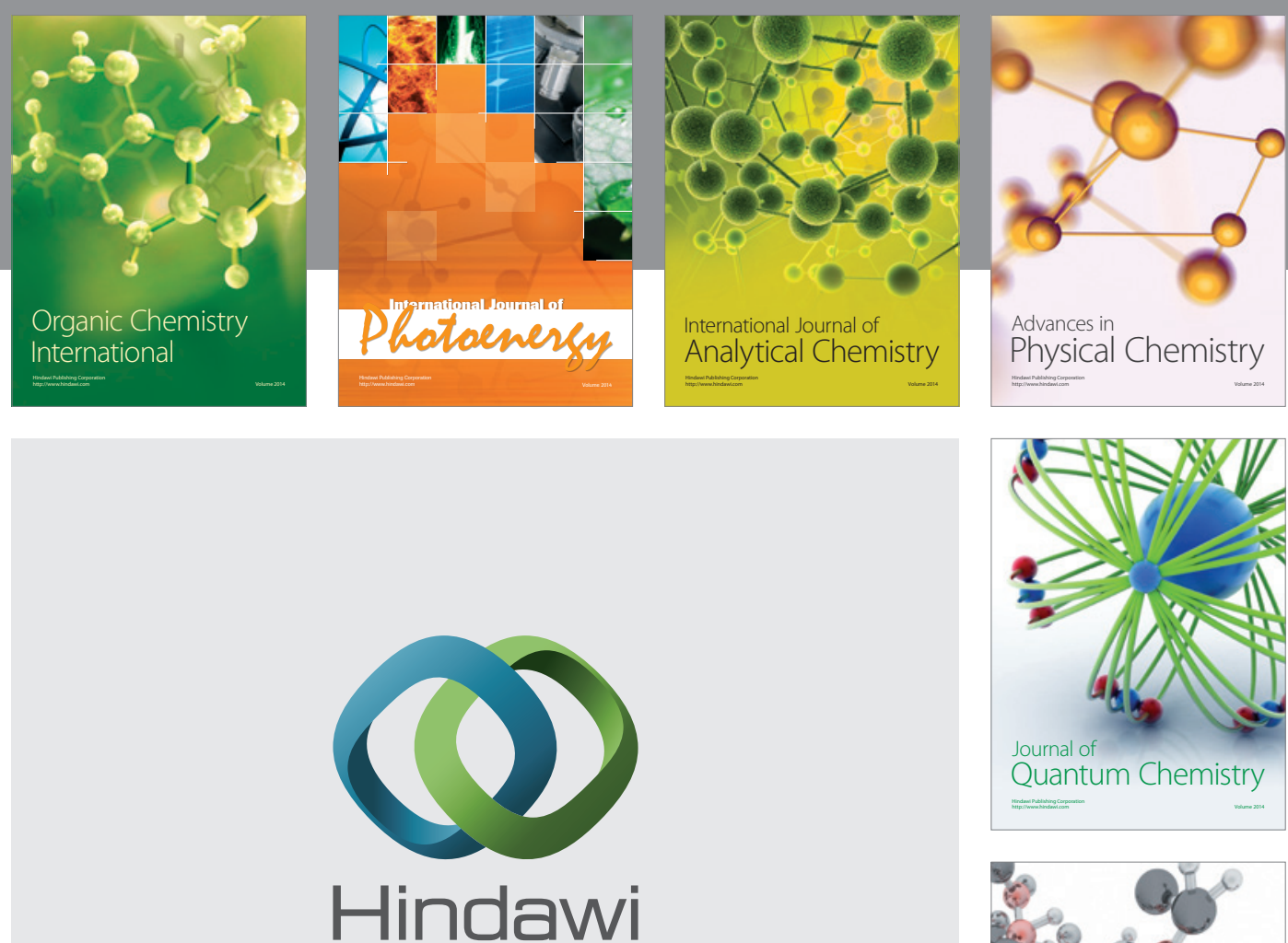

Submit your manuscripts at

http://www.hindawi.com

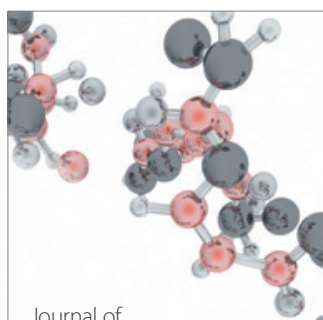

Analytical Methods

in Chemistry

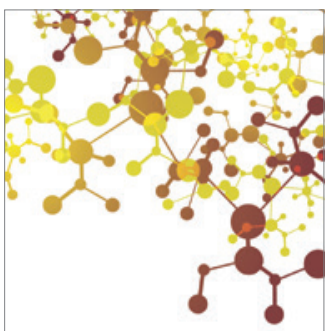

Journal of

Applied Chemistry

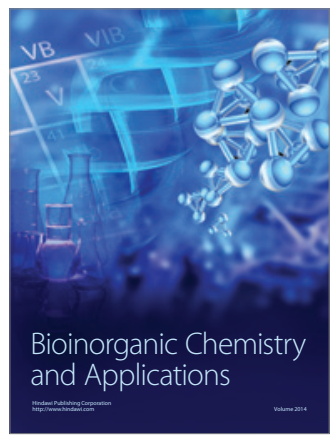

Inorganic Chemistry
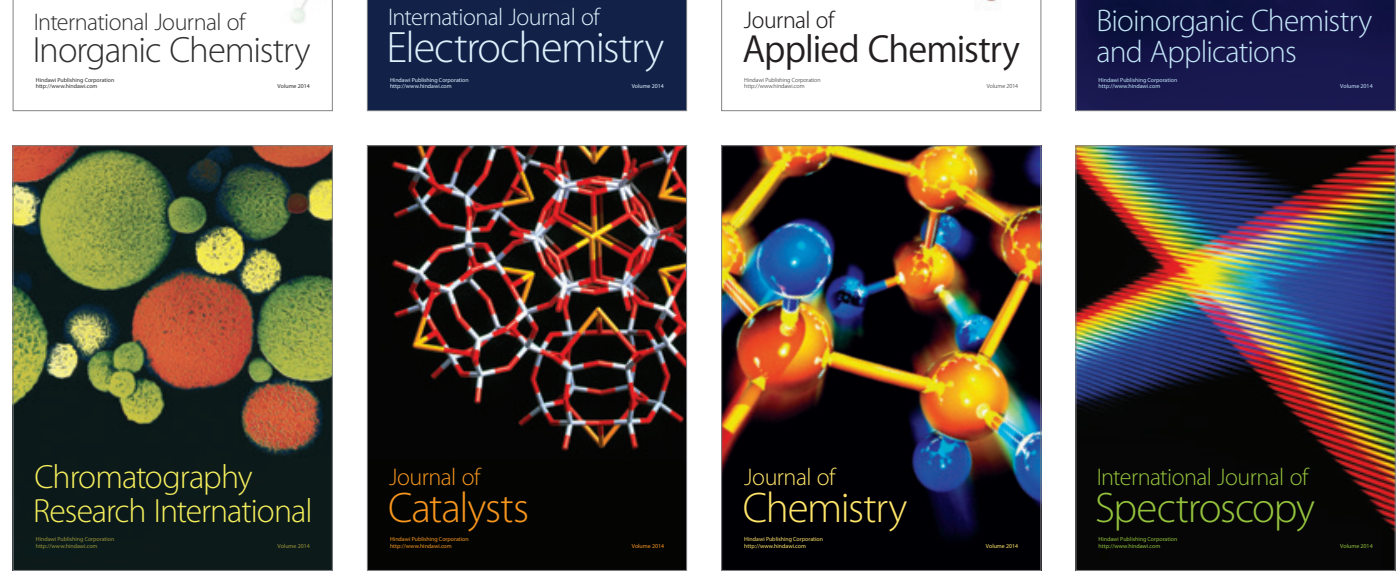